\title{
A Limited Sampling Schedule to Estimate Individual Pharmacokinetic Parameters of Fludarabine in Hematopoietic Cell Transplant Patients
}

\author{
David H. Salinger6 ${ }^{6}$, David K. Blough, Paolo Vicini ${ }^{7}$, Claudio Anasetti, Paul V. O’Donnell, \\ Brenda M. Sandmaier, and Jeannine S. McCune \\ University of Washington Departments of Bioengineering (DHS, PV), Pharmacy (DKB, JSM), and \\ Medicine (PVO, BMS). Fred Hutchinson Cancer Research Center (DKB, PVO, BMS, JSM). Both in \\ Seattle, WA. H. Lee Moffitt Cancer Center. Tampa, FL
}

\begin{abstract}
Purpose-Fludarabine monophosphate (fludarabine) is frequently administered to patients receiving a reduced-intensity conditioning regimen for allogeneic hematopoietic cell transplant (HCT) in an ambulatory care setting. These patients experience significant interpatient variability in clinical outcomes, potentially due to pharmacokinetic variability in 2-fluoroadenine (F-ara-A) plasma concentrations. To test such hypotheses, patient compliance with the blood sampling should be optimized by the development of a minimally intrusive limited sampling schedule (LSS) to characterize F-ara-A pharmacokinetics. To this end, we sought to create the first F-ara-A population pharmacokinetic model and subsequently a LSS.
\end{abstract}

Experimental Design-A retrospective evaluation of F-ara-A pharmacokinetics was conducted after one or more doses of daily IV fludarabine in 42 adult HCT recipients. NONMEM software was used to estimate the population pharmacokinetic parameters and compute the area under the concentration-time curve (AUC).

Results-A two compartment model best fit the data. A LSS was constructed using a simulation approach, seeking to minimize the scaled mean square error (sMSE) for the AUC for each simulated individual. The LSS times chosen were: 0.583 hour (hr), $1.5 \mathrm{hr}, 6.5 \mathrm{hr}$ and $24 \mathrm{hr}$ after the start of the 30 minute fludarabine infusion.

\footnotetext{
Corresponding author: Jeannine S. McCune, PharmD, University of Washington, Box 357630, Seattle WA 98195-7630, jmccune@u.washington.edu, 206-543-1412 (phone), 206-543-3835 (fax).

${ }^{6}$ Current address Amgen Inc., Seattle, Washington.

${ }^{7}$ Current address Pfizer Global Research and Development, La Jolla, CA

FOOTNOTES: None

ADDENDUM: None.

Statement of Translational Relevance

Fludarabine monophosphate (fludarabine) is a key component of many reduced-intensity conditioning regimens for allogeneic hematopoietic cell transplant (HCT). There is considerable variability in response and toxicity to fludarabine-containing conditioning regimens. Part of this variability in clinical outcomes may be due to pharmacokinetic variability in 2-fluoroadenine (F-ara-A) plasma concentrations. However, most patients receive fludarabine in an ambulatory care setting and thus the pharmacokinetic sampling must be minimally intrusive to facilitate adequate patient recruitment into pharmacodynamics studies. To this end, we developed a population pharmacokinetic model for F-ara-A disposition and evaluated covariates that contribute to its interpatient pharmacokinetic variability. We then determined a limited sampling schedule (LSS). The LSS is a critical tool for prospective studies evaluating if wide patient-topatient pharmacokinetic variability exists in patients receiving fludarabine and if such pharmacokinetic variability leads to clinically significant differences in efficacy or toxicity.
} 
Conclusion-The pharmacokinetics of F-ara-A in an individual HCT patient can be accurately estimated by obtaining 4 blood samples (using the LSS) and maximum a posteriori (MAP) Bayesian estimation.

Conclusions-These are essential tools for prospective pharmacodynamic studies seeking to determine if clinical outcomes are related to F-ara-A pharmacokinetics in patients receiving IV fludarabine in the ambulatory clinic.

\section{Keywords}

Fludarabine; nucleoside analogs; population pharmacokinetics; limited sampling schedule; hematopoietic cell transplant

\section{INTRODUCTION}

Allogeneic hematopoietic cell transplants (HCTs) have curative potential for patients with hematologic diseases and malignancies.(1,2) Myeloablative conditioning regimens, however, have high rates of non-relapse mortality, thus limiting their availability to older or medically infirm patients.(1-3) These patients can now receive an HCT with a reduced-intensity conditioning regimen. $(4,5)$ Administered intravenously as a more soluble pro-drug fludarabine monophosphate (Fludara ${ }^{\circledR}$, abbreviated with the term 'fludarabine' for the rest of the manuscript), the purine nucleoside analog fludarabine is administered at doses ranging from $90-250 \mathrm{mg} / \mathrm{m}^{2}$ in reduced-intensity HCT conditioning regimens.(2) Fludarabine administration depletes lymphocytes and improves engraftment rates (versus total body irradiation (TBI) alone), but it rarely causes fatal non-hematologic toxicity. $(2,5,6)$ Identification of the optimal fludarabine dose - one that achieves the delicate balance between engraftment rates and toxicity - would be of significant benefit to patients receiving nonmyeloablative HCT.

After administration, fludarabine is quickly dephosphorylated to F-ara-A by nucleotidases. (7-9) F-ara-A subsequently undergoes uptake and sequential phosphorylation to its active metabolite, fludarabine triphosphate (F-ara-ATP), which inhibits ribonucleotide reductase and DNA polymerase.(8) The toxicity to fludarabine monotherapy is not predicted by body surface area (BSA)-based dosing;(10) however, an inverse relationship has been observed between plasma F-ara-A AUC and neutropenia.(11) Preliminary data from patients receiving fludarabine as part of myeloablative HCTs suggest conditioning regimen-dependent pharmacodynamic relationships with F-ara-A AUC. $(12,13)$ Understanding the sources of variability in nonmyeloablative HCT patients is of the utmost importance, because HCT's success is based on achieving a delicate balance between the donor's and recipient's immune systems. The pharmacokinetic variability in F-ara-A may contribute to this narrow balance between the donor and recipient; however, this hypothesis has yet to be tested. Because fludarabine is administered in an ambulatory care clinic, it is critical to develop a limited sampling schedule (LSS) to facilitate patient compliance with the pharmacokinetic sampling necessary to evaluate the pharmacodynamics of F-ara-A in nonmyeloablative HCT recipients. To this end, we sought to develop a population pharmacokinetic model and LSS to minimize the patient burden for participation in pharmacodynamic studies to evaluate if $\mathrm{F}$-ara-A exposure predicts outcomes. Future pharmacodynamic studies are essential for determining the relevance of the F-ara-A pharmacokinetic variability upon clinical outcomes in outpatient nonmyeloablative HCT recipients. 


\section{MATERIALS \& METHODS}

\section{Patient population}

Forty-two patients received myeloablative conditioning with busulfan and fludarabine. The busulfan doses were personalized to achieve a target $\left({ }^{\mathrm{T}} \mathrm{BU}\right)$ concentration at steady state of $800-1000 \mathrm{ng} / \mathrm{ml}$. Fludarabine doses were not changed based on F-ara-A pharmacokinetic data. F-ara-A pharmacokinetic data were available on 27 patients receiving targeted oral busulfan for 4 days followed by fludarabine ( $30 \mathrm{mg} / \mathrm{m}^{2} /$ day for 4 days) (Protocol \#1519).(13) F-ara-A pharmacokinetic data were available in 15 additional patients enrolled on a subsequent ${ }^{\mathrm{T}} \mathrm{BU} /$ fludarabine protocol (Protocol \#2041). This regimen consisted of fludarabine $50 \mathrm{mg} / \mathrm{m}^{2} / \mathrm{day}$ for 5 days (days -6 to -2 ), targeted daily intravenous (IV) busulfan on days -5 to -2 , and thymoglobulin on days -3 to -1 . In both regimens, fludarabine was infused through a central venous access catheter over 30 minutes. Institutional Review Board approval was obtained prior to study conduct and all patients or patient guardians gave informed consent before their participation in the study began.

All patients had been diagnosed with hematologic disorders and had adequate renal (i.e., serum creatinine $<1.5 \mathrm{mg} / \mathrm{dL}$, and creatinine clearance or radioisotope glomerular filtration rate $>60$ $\mathrm{ml} / \mathrm{min} / 1.73 \mathrm{~m}^{2}$ ) and liver (i.e., total bilirubin $<1.5 \mathrm{mg} / \mathrm{dL}$ and alanine aminotransferase $<300$ units/L) function. The body weight and BSA used to calculate doses were based on institutional guidelines.

We evaluated demographic data, concomitant medications and relevant laboratory tests for 3 days before and during fludarabine administration to identify potential covariates. Potential covariates included: age at the time of fludarabine administration, gender, BSA, actual body weight, adjusted ideal body weight, height, serum creatinine, creatinine clearance (estimated using Cockcroft-Gault formula),(14) blood urea nitrogen, and albumin. Patients received similar antiemetics, antibiotics and antifungals per institutional Standard Practice Guidelines; corticosteroids were not used as antiemetics with fludarabine. Genetic covariates were not evaluated because fludarabine is mainly renally eliminated.(11,15,16)

\section{Fludarabine pharmacokinetic sampling and analysis}

F-ara-A pharmacokinetic blood samples were drawn from a catheter not used to infuse the fludarabine dose. In the 27 Protocol \#1519 patients who received fludarabine $30 \mathrm{mg} / \mathrm{m}^{2} / \mathrm{day}$, pharmacokinetic blood samples were scheduled to be drawn immediately before and at the end of the 30 minute infusion $(0.5 \mathrm{hr})$, and $1 \mathrm{hr}, 4 \mathrm{hr}, 8 \mathrm{hr}, 12 \mathrm{hr}, 24 \mathrm{hr}$ after the start of the infusion. The $24 \mathrm{hr}$ pharmacokinetic sample was drawn before the next dose. These samples were collected after all four fludarabine doses. The F-ara-A pharmacokinetic data from this study was previously reported.(13) Outpatient fludarabine administration necessitated changing the pharmacokinetic sampling times in the subsequent study (i.e., Protocol \#2041). In the 15 Protocol \#2041 patients who received fludarabine $50 \mathrm{mg} / \mathrm{m}^{2} /$ day, pharmacokinetic samples were drawn at $0.5 \mathrm{hr}, 5 \mathrm{~min}$ after the end of the infusion $(0.583 \mathrm{hr}), 1.5 \mathrm{hr}, 4.5 \mathrm{hr}, 6.5 \mathrm{hr}$, and $24 \mathrm{hr}$ after the start of the infusion. The $24 \mathrm{hr}$ pharmacokinetic sample was drawn before the next dose. Pharmacokinetic samples were collected with the first fludarabine dose only (i.e., day -6) in Protocol \#2041 patients.

F-ara-A plasma concentrations were quantitated using LC/MS as previously described;(13) the dynamic range was 0.23 to $9.04 \mu \mathrm{M}$ and the interday $\mathrm{CV}$ was $<10 \%$.

\section{Population pharmacokinetic modeling}

Seven hundred twenty eight (728) concentration-time points were available for population pharmacokinetic modeling. Concomitant busulfan does not interact with F-ara-A(17) and 
therefore, the datasets were combined for the population pharmacokinetic modeling and construction of the LSS. The pharmacokinetic model of F-ara-A was fit using the first-order method in the nonlinear mixed-effects modeling software NONMEM (version VI).(18) A two compartment model of F-ara-A was initially tested to estimate the following parameters: CL, the apparent elimination clearance; V1, the apparent volume of distribution in plasma; Q, the inter-compartmental clearance; and V2, the apparent volume of the peripheral compartment. Between-subject variability (BSV) of parameters was initially modeled using a full covariance matrix; other alternative covariance matrix structures were tested as well. We assumed a lognormal distribution of BSV for all parameters. Residual unknown variability (RUV) was estimated with a combination of additive and proportional error models. Individual parameters were calculated as empirical Bayes estimates using the POSTHOC option in NONMEM. Criteria for model selection included visual inspection of goodness of fit plots (data vs. population and individual predictions), residuals, and weighted residuals. Plots of data vs. population and individual predictions, data vs. residuals and data vs. weighted residuals were visually inspected for goodness of fit. Models with different numbers of parameters were selected based on expected differences in chi-square distributions with appropriate degrees of freedom.(19)

Subsequently, we evaluated if the model was improved by inclusion of the following covariates in the base pharmacokinetic model: age, gender, BSA, weight (actual or adjusted ideal body weight), height, creatinine, creatinine clearance, blood urea nitrogen, and albumin. Linear regression relating individual parameter values and covariates, as well as visual inspection of plots, were used to explore these relationships. Inclusion/exclusion of covariates was determined by parsimony criteria.

As a validation of the model, a visual predictive check was performed.(20) We simulated data for 1000 subjects using the final population pharmacokinetic model with BSA-normalized dosing (see Results for a rationale for BSA-normalized dosing). The median and $90 \%$ prediction intervals were plotted with the original data.

\section{Determination of optimal limited sampling schedule (LSS) via simulation}

We sought to choose an optimal LSS that balanced the practical challenges of pharmacokinetic sampling in patients with the need for sufficient data to predict an individual's F-ara-A AUC. Specific challenges were that the LSS had to have few enough samples to encourage patient compliance (chosen as a maximum of four pharmacokinetic samples) and a short enough time span that patients can receive fludarabine in an ambulatory clinic (i.e., within 6.5 hours from the start of the infusion). Therefore, three of the four LSS times were chosen within the first 6.5 hours after the start of the fludarabine infusion. The fourth LSS was set at $24 \mathrm{hr}$, which is immediately prior to the next fludarabine dose. Individual pharmacokinetic parameters were determined via maximum a posteriori (MAP) Bayesian estimation, where the population prior (from the population pharmacokinetic analysis) may potentially offset the loss of individual data due to the use of the LSS.

We hierarchically simulated 2000 subjects whose pharmacokinetic parameters were based on our population pharmacokinetic model of F-ara-A after IV fludarabine administration. Each subject arose from the model BSV and was replicated 20 times using different simulated RUV (data error) instantiations. For each potential LSS, we performed individual MAP parameter estimation for each subject's simulated data for each error replicate, and computed both the 24 $\mathrm{hr}$ and $120 \mathrm{hr}$ AUCs after one simulated fludarabine infusion. The modeled AUCs were compared to the true (simulated) AUCs to compute scaled mean squared error (sMSE). The mean (across subjects) sMSE was the figure of merit for each LSS. The LSS with minimum sMSE was considered optimal. For each simulated subject, the mean squared error: 
$M S E=\left(\frac{1}{N} \sum_{i=1}^{N}\left(\widehat{\theta}_{i}-\bar{\theta}\right)\right)^{2}+\frac{1}{N} \sum_{i=1}^{N}\left[\left(\widehat{\theta}_{i}-\bar{\theta}\right)-\frac{1}{N} \sum_{i=1}^{N}\left(\widehat{\theta}_{i}-\bar{\theta}\right)\right]^{2}$ and scaled MSE: $s M S E=$ $M S E / \theta^{2}$ were computed for the AUC for each potential time grid, where $\hat{\theta}_{i}$ is the AUC for error replicate $i$, and $\theta$ is the "true" AUC value.

To determine how the LSS would perform in the calculation of the AUC compared to the intensive, full pharmacokinetic sampling (FULL, at 0.5, 0.583, 1.5, 4.5, 6.5 and $24 \mathrm{hr}$ post dose) - where all 6 concentration-time data points were fit to the model via individual maximum likelihood estimation (MLE; i.e. without parameter prior) - the same subjects were also considered and the root mean sMSE computed for FULL.

The framework to accomplish this LSS optimization was constructed using the R statistical software, with the needed simulation and parameter estimation performed via system calls to NONMEM.

\section{RESULTS}

\section{Patient population}

Of the 42 patients studied, 21 were male and 21 were female. At the time of fludarabine administration, the median age was 49.1 years (range: 12.6 - 65.5), the median BSA (based on adjusted ideal body weight) was $1.81 \mathrm{~m}^{2}$ (range: $1.51-2.14$ ), and the actual body weight was $85.1 \mathrm{~kg}$ (range: 55.4 - 139.7). Creatinine clearance $(\mathrm{N}=40)$ was $91 \mathrm{ml} / \mathrm{min}$ (range: 55 - 148).

\section{Population pharmacokinetic modeling}

We determined that the best population pharmacokinetic model for these data was a first-order pharmacokinetic, two-compartment model with a 30 minute fludarabine infusion as an input to the central compartment. Comparison of a full covariance matrix BSV to various other configurations indicated that the correlations between CL (apparent elimination clearance) and $\mathrm{Q}$ (the intercompartmental clearance) and CL and V2 (apparent volume of the peripheral compartment) were not significantly $(\mathrm{p}<0.05)$ different from zero. All covariance configurations confirmed a very strong correlation (near 1) between Q and V2. Estimation of a correlation near one can lead to numerical instabilities and may disrupt determination of convergence. Thus, for the final base model, we concluded that the best BSV covariance structure was a banded covariance matrix (implying that $\operatorname{corr}(\mathrm{CL}, \mathrm{Q})=\operatorname{corr}(\mathrm{CL}, \mathrm{V} 2)=0)$, with the additional assumption that the correlation of $\mathrm{Q}$ and $\mathrm{V} 2$ was fixed to 1 . Also, by this construction, $\operatorname{corr}(\mathrm{V} 1, \mathrm{~V} 2)=\operatorname{corr}(\mathrm{V} 1, \mathrm{Q})$. More detail about this covariance structure and its formulation in NONMEM is provided in Appendix 1. A combined additive and proportional RUV error model performed better $(\mathrm{p}<0.05)$ than either an additive or a proportional error model alone.

Visual inspection of plots and linear regression relating individual parameter and covariate values revealed the strongest association between all parameters (i.e., CL, V1, Q, V2) and the various measures of body size (i.e., BSA, height, actual body weight, adjusted ideal body weight). Since the body size measures (except height) were highly correlated, we chose BSA because fludarabine is dosed based on BSA (e.g., $30 \mathrm{mg} / \mathrm{m}^{2} /$ day). As a first step, we evaluated BSA-normalized dosing, essentially testing BSA as a covariate (scaling) of all parameters. This inclusion was justified by a 13.3 point improvement in objective function value with no additional parameters and a 4 percentage point decrease in BSV for each parameter. The structural parameters fixed effects for this BSA-normalized dosing are provided in Table 1. The right half of Table 1 presents the BSV of the model parameters as the lower half of a matrix, with variability $(\% \mathrm{CV})$ on the diagonal and parameter correlation (as Pearson $\mathrm{r}$ ) in the off-diagonal elements. 
Using the BSA-normalized dosing model, visual inspection of plots and linear regression relating individual parameter and covariate values revealed weak relationships between V1 and gender and both CL and V1 and height. These were added separately as linear parametercovariate relationships to the BSA-normalized model. Inclusion of V1 as a linear model in terms of height and (separately) of gender did not reach statistical significance ( $<<0.05$; objective function improvement of <3.84).(19)

The inclusion of CL as a linear model of height did reach the required $p<0.05$ level of statistically significant objective function improvement (the decrease was 5.5), although it was not strongly significant (i.e. not at the $\mathrm{p}<0.01$ level, which would require an objective function decrease of 6.6). The typical CL value of $5.70 \mathrm{~L} / \mathrm{hr} / \mathrm{m}^{2}$ (Table 1 ) was replaced by $5.74+0.0334 *$ (height-170.5) where height is the subject's height and the median height was $170.5 \mathrm{~cm}$. This inclusion also resulted in the explanation of 4.2 percentage points of BSV in CL. Other parameter and variability values changed only negligibly.

Figures 1 and 2 provide model validation in the form of a visual predictive check. The median and $90 \%$ prediction intervals were plotted with the original data for subjects receiving IV fludarabine $30 \mathrm{mg} / \mathrm{m}^{2} /$ day with pharmacokinetic sampling after all four doses (Figure 1) and IV fludarabine $50 \mathrm{mg} / \mathrm{m}^{2} /$ day with pharmacokinetic sampling after the first dose only (Figure 2). The visual predictive check shows generally good agreement between model and data, although somewhat fewer than $10 \%$ of data points lie outside the $90 \%$ prediction interval.

\section{Determination of optimal LSS via simulation}

LSS results are presented in Table 2 ranked by root mean sMSE for each potential LSS (so that the units are in percent standard deviation). A comparison of schedules based on root mean sMSE for the $24 \mathrm{hr}$ and $120 \mathrm{hr}$ AUC showed a number of schedules performing similarly well (Tables 2 and 3). We chose the 0.583, 1.5, 6.5 and $24 \mathrm{hr}$ LSS as the optimal schedule. This decision was based on the schedule's low sMSE and on our experience with patient compliance with pharmacokinetic sampling. Other potential schedules performed nearly as well. The performance of the FULL schedule (6 observations; model fit by MLE parameter estimation, i.e. no population prior) was nearly as good as the chosen LSS for determining the $24 \mathrm{hr}$ AUC, but performed worse than the LSS for determining the $120 \mathrm{hr}$ AUC.

\section{DISCUSSION}

This work represents a critical step for future F-ara-A pharmacodynamic studies in patients receiving fludarabine in the ambulatory clinic, in which the pharmacokinetic scheduling must be convenient enough to ensure patient compliance while still accurately characterizing an individual's pharmacokinetic disposition of F-ara-A. To this goal, we developed a novel population pharmacokinetic model and determined a LSS. This minimally intrusive LSS, when used in conjunction with population prior values, performs as well as (Table 2) or better than (Table 3) more intense pharmacokinetic sampling with the individual's pharmacokinetic data alone using compartmental analysis (MLE). The LSS chosen to characterize an individual's F-ara-A pharmacokinetics - when used in combination with population pharmacokinetic priors - was $0.583 \mathrm{hr}, 1.5 \mathrm{hr}, 6.5 \mathrm{hr}$ and $24 \mathrm{hr}$ after the start of the $0.5 \mathrm{hr}$ fludarabine infusion.

In a subsequent investigation, we are employing this LSS to test the hypothesis that plasma Fara-A AUC is associated with day $28 \mathrm{~T}$-cell donor chimerism in patients receiving fludarabine/ TBI conditioning in an outpatient setting. Day $28 \mathrm{~T}$-cell chimerism is associated with engraftment, relapse, and acute graft-versus-host disease rates, $(2,5,6)$, and therefore we seek to determine if F-ara-A AUC is associated with day 28 chimerism. As fludarabine/TBI is one of the most common reduced-intensity regimens, it is imperative to identify biomarkers associated with donor chimerism in these nonmyeloablative HCT recipients.(21) The 
availability of HCT has expanded due to the recent development of reduced-intensity conditioning regimens. $(2,22)$ With the current method dosing of fludarabine (i.e., based on BSA), there is substantial variability in donor chimerism, efficacy and toxicity in nonmyeloablative HCT recipients. We hypothesize that this interpatient variability in donor chimerism may be related to interpatient variability in F-ara-A pharmacokinetics. We are the first to develop a population pharmacokinetic model of F-ara-A in HCT recipients and determine a LSS which requires only four blood samples to estimate an individual's F-ara-A AUC when used in combination with population parameter priors. With the development of these critical tools, we are testing the hypothesis that F-ara-A AUC is associated with clinical outcomes in patients receiving nonmyeloablative HCT conditioning in the ambulatory clinic.

The interpatient variability in F-ara-A clearance is comparable to that of busulfan, the dosing of which is personalized based on pharmacokinetics at many HCT centers.(23) It is critical to establish if the pharmacokinetic variability in F-ara-A AUC is associated with clinical outcomes and if so, subsequently conduct a clinical trial to demonstrate the clinical benefit of personalized dosing. Our group has taken that approach with both busulfan(24) and cyclophosphamide. $(25,26)$ With both these alkylating agents, the pharmacodynamics differs based on the conditioning regimens. Preliminary data suggest conditioning regimen-dependent pharmacodynamic relationships with F-ara-A AUC. In patients receiving busulfan/fludarabine myeloablative conditioning, F-ara-A AUC was not related to T-cell chimerism or engraftment. (13) However, in patients receiving fludarabine in combination with cyclophosphamide and total body irradiation, those with a low F-ara-A AUC were less likely to experience neutrophil engraftment while the patient with the highest F-ara-A AUC died due to neurotoxicity.(12) Therefore, future studies testing the hypothesis that variable clinical outcomes are associated with F-ara-A AUC should be conducted in a homogenous group of nonmyeloablative recipients all of which are receiving fludarabine/TBI. With fludarabine-containing conditioning regimens, the development of this population pharmacokinetic model and LSS provide critical tools to evaluate if F-ara-A AUC is associated with clinical outcomes.(2,21,27-29) The LSS requiring four blood samples is minimally intrusive to HCT patients treated in the ambulatory clinic, while still allowing for accurate characterization of F-ara-A AUC to be used in our ongoing pharmacodynamic study in nonmyeloablative conditioned patients receiving fludarabine/TBI.

In conjunction with characterizing F-ara-A AUC, we seek to also evaluate the role of fludarabine triphosphate (F-ara-ATP) in donor chimerism. After administration, fludarabine is quickly dephosphorylated to F-ara-A by nucleotidases.(7-9) F-ara-A subsequently undergoes uptake, and is then sequentially phosphorylated to its monophosphate, diphosphate, and triphosphate metabolites.(8) The inhibition of ribonucleotide reductase and DNA polymerase by fludarabine triphosphate (F-ara-ATP), the active moiety, ultimately leads to cellular apoptosis.(8) We have recently created a novel method to phenotype F-ara-ATP accumulation in $\mathrm{CD}^{+}$and $\mathrm{CD} 8^{+}$cells.(30) F-ara-ATP accumulation - used in conjunction with F-ara-AUC - can be used to estimate F-ara-ATP concentrations in $\mathrm{CD}^{+}$and $\mathrm{CD} 8^{+}$cells.

This population pharmacokinetic model is the first to be constructed for F-ara-A plasma pharmacokinetics. The model estimates of clearance agree with previously reported data analyzed with noncompartmental or compartmental pharmacokinetic analysis in cancer patients not undergoing an allogeneic HCT.(11,15-17) Therefore, with further validation, this population pharmacokinetic model and LSS may be used in non-transplant patients receiving IV fludarabine in the ambulatory clinic. Outside of the setting of allogeneic HCT, fludarabine is used to treat patients with chronic lymphocytic leukemia, follicular lymphoma, or acute myeloid leukemia. $(9,31,32)$ In patients receiving fludarabine monotherapy, an inverse relationship has been observed between plasma F-ara-A AUC and neutropenia.(11) It is also of interest to determine if elevated F-ara-A AUC is associated with neurotoxicity outside the 
setting of allogeneic HCT,(12) which is particularly important because neurotoxicity was doselimiting in phase I studies of fludarabine monotherapy.(7) Therefore, the development of this population pharmacokinetic model and LSS provide tools for future studies to test the hypothesis that elevated F-ara-A AUC is related to toxicity outside the setting of allogeneic HCT.(12)

Population pharmacokinetic analysis provides a rigorously quantitative approach to incorporating population information (i.e., typical values and variability of the disposition of drugs through the body) in the determination of parameter priors which can assist in estimation of an individual's exposure (via MAP Bayesian estimation). The benefits of population pharmacokinetic modeling are just starting to be realized in HCT recipients, as evidenced by our results with cyclophosphamide. $(33,34)$ This population pharmacokinetic model is the first, to our knowledge, to be built for F-ara-A. It was built using F-ara-A concentration-time data on days that only fludarabine - not busulfan or thymoglobulin - was administered to patients conditioned with myeloablative doses of ${ }^{\mathrm{T}} \mathrm{BU} / \mathrm{FLU}$. Notably, concomitant busulfan does not interact with F-ara-A pharmacokinetics.(17) A limitation of this F-ara-A dataset was that all patients had adequate renal and liver function to receive myeloablative ${ }^{\mathrm{T}} \mathrm{BU} / \mathrm{FLU}$. However, some nonmyeloablative HCT recipients may have impaired renal or liver function. Notably, none of the indices of renal or liver function were covariates in our analysis (See 'Population Pharmacokinetic Modeling' of Results section). An improved understanding of the covariates (e.g., age) of BSV can lead to improved experimental design and determination of a LSS.

(35) BSA was the only covariate included in the model. The inclusion of subject height as a covariate for apparent clearance (CL) was found to barely reach statistical significance $(\mathrm{p}<0.05)$; our opinion is that inclusion of this relationship in the model will likely not have clinical impact. We observed a very strong correlation of parameters Q and V2 in our model. To account for this correlation, we tried alternative covariance matrix structures which did allow us to fix a correlation to less than 1, but these required a block-diagonal covariance matrix which assumed a zero correlation between parameters V1 and both Q and V2. These alternative covariance structures were determined (by parsimony criteria) to be inferior to our final model. Also, fixing $\operatorname{corr}(\mathrm{Q}, \mathrm{V} 2)$ to, say, 0.98 seemed as arbitrary as fixing the correlation to 1 .

Therefore, although it is unlikely that two model parameters will have an actual correlation of 1 among subjects, we felt this assumption was justified. Pertinent to the creation of the LSS, while D-optimality is the customary optimality criterion in population experimental design, we opted to use MSE in this individual experimental design setting. D-Optimality, the determinant of the Fisher Information Matrix, accounts for parameter precision only and ignores estimator bias. We expect our individual MAP estimates to be biased due to the presence of the population prior.(36) Using MSE accounts for both estimator precision and bias.(37)

In conclusion, our successful development of the first population pharmacokinetic model and LSS to characterize F-ara-A AUC represents an essential step in executing future pharmacodynamic studies patients receiving fludarabine in an ambulatory clinic. This minimally intrusive LSS will allow us to test the hypothesis that F-ara-A AUC - estimated through the use of this four-sample LSS and population pharmacokinetic priors - is associated with donor chimerism and clinical outcomes in a homogenous population of patients receiving nonmyeloablative conditioning of IV fludarabine/TBI.

\section{ACKNOWLEDGEMENTS}

We are grateful to the study participants, Terry Furlong for his assistance with collection of the F-ara-A pharmacokinetic data, and Meagan Bemer for her assistance with database management and manuscript preparation. We also greatly appreciate the insightful comments of Rainer Storb, MD, to an earlier version of this manuscript. 


\section{Appendix 1. The base pharmacokinetic model was implemented in NONMEM}

Implementation of the assumed $\operatorname{Cor}(\mathrm{Q}, \mathrm{V} 2)=1$ is reported below; as well as some notes of explanation.

The model parameterizations and lognormal BSV are formulated as:

$$
\begin{aligned}
& \mathrm{CL}=\operatorname{THETA}(1) * \operatorname{EXP}(\operatorname{ETA}(1)) \\
& \mathrm{V}_{1}=\operatorname{THETA}(2) * \operatorname{EXP}(\operatorname{ETA}(2)) \\
& \mathrm{Q}=\operatorname{THETA}(3) * \operatorname{EXP}(\operatorname{ETA}(3)) \\
& \mathrm{V}_{2}=\operatorname{THETA}(4) * \operatorname{EXP}(\operatorname{ETA}(3) * \operatorname{THETA}(5))
\end{aligned}
$$

Notes:

$$
\begin{aligned}
& \operatorname{Cov}\left(C L, V_{1}\right)=\theta_{1} \theta_{2} \operatorname{Cov}\left(\eta_{1}, \eta_{2}\right) \\
& \operatorname{Corr}\left(C L, V_{1}\right)=\operatorname{Corr}\left(\eta_{1}, \eta_{2}\right)
\end{aligned}
$$

By construction, the correlation $\operatorname{Corr}(\mathrm{Q}, \mathrm{V} 2)=1$ :

$$
\begin{aligned}
X & =\eta_{3}, Y=\eta_{3} \theta_{5} \\
\operatorname{Var}(X) & =\operatorname{Var}\left(\eta_{3}\right) \\
\operatorname{Var}(Y) & =\theta_{5}^{2} \operatorname{Var}\left(\eta_{3}\right) \\
\operatorname{Cov}(X, Y) & =E[X Y]-E[X] E[Y]=E[X Y] \\
& =\theta_{5} E\left[\eta_{3}^{2}\right] \\
& =\theta_{5} \operatorname{Var}\left[\eta_{3}\right] \\
\operatorname{Corr}(X, Y) & =\frac{\operatorname{Cov}(X, Y)}{\sqrt{\operatorname{Var}(X) \operatorname{Var}(Y)}}=1 \\
& \text { Similarly, } \\
\operatorname{Corr}\left(\eta_{2}, \eta_{3} \theta_{5}\right) & =\operatorname{Corr}\left(\eta_{2}, \eta_{3}\right)
\end{aligned}
$$

$\theta_{5}$ can be interpreted as the (square root of the) scale parameter between $\operatorname{Var}(\mathrm{Q})$ and $\operatorname{Var}$ $\left(\mathrm{V}_{2}\right)$.

The value of $\theta_{5}$ corresponding to the parameter values in Table 1 is 1.04 (11.4\% SE).

\section{REFERENCES}

1. Copelan EA. Hematopoietic stem-cell transplantation. N Engl J Med 2006;354:1813-26. [PubMed: 16641398]

2. Deeg HJ, Maris MB, Scott BL, Warren EH. Optimization of allogeneic transplant conditioning: not the time for dogma. Leukemia 2006;20:1701-5. [PubMed: 16888618]

3. Baron F, Storb R, Little MT. Hematopoietic cell transplantation: five decades of progress. Arch Med Res 2003;34:528-44. [PubMed: 14734093]

4. Aoudjhane M, Labopin M, Gorin NC, et al. Comparative outcome of reduced intensity and myeloablative conditioning regimen in HLA identical sibling allogeneic haematopoietic stem cell transplantation for patients older than 50 years of age with acute myeloblastic leukaemia: a retrospective survey from the Acute Leukemia Working Party (ALWP) of the European group for Blood and Marrow Transplantation (EBMT). Leukemia 2005;19:2304-12. [PubMed: 16193083]

5. Maris MB, Niederwieser D, Sandmaier BM, et al. HLA-matched unrelated donor hematopoietic cell transplantation after nonmyeloablative conditioning for patients with hematologic malignancies. Blood 2003;102:2021-30. [PubMed: 12791654]

6. Mielcarek M, Burroughs L, Leisenring W, et al. Prognostic relevance of 'early-onset' graft-versushost disease following non-myeloablative haematopoietic cell transplantation. Br J Haematol 2005;129:381-91. [PubMed: 15842663] 
7. Danhauser L, Plunkett W, Liliemark J, Gandhi V, Iacoboni S, Keating M. Comparison between the plasma and intracellular pharmacology of 1-beta-D-arabinofuranosylcytosine and 9-beta-Darabinofuranosyl-2-fluoroadenine 5'-monophosphate in patients with relapsed leukemia. Leukemia 1987;1:638-43. [PubMed: 3478543]

8. Gandhi V, Plunkett W. Cellular and clinical pharmacology of fludarabine. Clin Pharmacokinet 2002;41:93-103. [PubMed: 11888330]

9. Robak T, Lech-Maranda E, Korycka A, Robak E. Purine nucleoside analogs as immunosuppressive and antineoplastic agents: mechanism of action and clinical activity. Current medicinal chemistry 2006;13:3165-89. [PubMed: 17168705]

10. Seymour L, Eisenhauer E. A review of dose-limiting events in phase I trials: antimetabolites show unpredictable relationships between dose and toxicity. Cancer Chemother Pharmacol 2001;47:2-10. [PubMed: 11221956]

11. Hersh MR, Kuhn JG, Phillips JL, Clark G, Ludden TM, Von Hoff DD. Pharmacokinetic study of fludarabine phosphate (NSC 312887). Cancer Chemother Pharmacol 1986;17:277-80. [PubMed: 2427240]

12. Jacobson PA, Rogosheske J, Green K, et al. Fludarabine Pharmacokinetics in Nonmyeloablative Hematopoietic Cell Transplantation (HCT): Association with Engraftment and Neurotoxicty. Blood 2005;106Abstract \#3673

13. Bornhauser M, Storer B, Slattery JT, et al. Conditioning with fludarabine and targeted busulfan for transplantation of allogeneic hematopoietic stem cells. Blood 2003;102:820-6. [PubMed: 12676781]

14. Cockcroft DW, Gault MH. Prediction of creatinine clearance from serum creatinine. Nephron 1976;16:31-41. [PubMed: 1244564]

15. Plunkett W, Gandhi V, Huang P, et al. Fludarabine: pharmacokinetics, mechanisms of action, and rationales for combination therapies. Semin Oncol 1993;20:2-12. [PubMed: 8235690]

16. Malspeis L, Grever MR, Staubus AE, Young D. Pharmacokinetics of 2-F-ara-A (9-beta-Darabinofuranosyl-2-fluoroadenine) in cancer patients during the phase I clinical investigation of fludarabine phosphate. Semin Oncol 1990;17:18-32. [PubMed: 1699279]

17. Bonin M, Pursche S, Bergeman T, et al. F-ara-A pharmacokinetics during reduced-intensity conditioning therapy with fludarabine and busulfan. Bone Marrow Transplant 2007;39:201-6. [PubMed: 17211431]

18. Beal, SL.; Sheiner, LB.; Boeckmann, AJ., editors. NONMEM Users Guides. Icon Development Solutions; Ellicott City: 19892006. NONMEM Users Guides1989-2006

19. Wahlby U, Jonsson EN, Karlsson MO. Assessment of actual significance levels for covariate effects in NONMEM. J Pharmacokinet Pharmacodyn 2001;28:231-52. [PubMed: 11468939]

20. Holford, NHG. The Visual Predictive Check - Superiority to Standard Diagnostic (Rorschach) Plots; Abstracts of the Annual Meeting of the Population Approach Group in Europe (PAGE); 2005; Abstr 738

21. Giralt S, Logan B, Rizzo D, et al. Reduced-intensity conditioning for unrelated donor progenitor cell transplantation: long-term follow-up of the first 285 reported to the national marrow donor program. Biol Blood Marrow Transplant 2007;13:844-52. [PubMed: 17580263]

22. Mielcarek M, Sandmaier BM, Maloney DG, et al. Nonmyeloablative hematopoietic cell transplantation: status quo and future perspectives. Journal of clinical immunology 2002;22:70-4. [PubMed: 11998895]

23. McCune JS, Gibbs JP, Slattery JT. Plasma concentration monitoring of busulfan: does it improve clinical outcome? Clin Pharmacokinet 2000;39:155-65. [PubMed: 10976660]

24. Slattery JT, Sanders JE, Buckner CD, et al. Graft-rejection and toxicity following bone marrow transplantation in relation to busulfan pharmacokinetics. Bone Marrow Transplant 1995;16:31-42. [PubMed: 7581127]

25. McDonald GB, Slattery JT, Bouvier ME, et al. Cyclophosphamide metabolism, liver toxicity, and mortality following hematopoietic stem cell transplantation. Blood Mar 1;2003 101:2043-8. [PubMed: 12406916]

26. McCune J, Batchelder A, Guthrie K, et al. Personalized Dosing of Cyclophosphamide in the Total Body Irradiation-Cyclophosphamide Conditioning Regimen: A Phase II Trial in Patients With Hematologic Malignancy. Clin Pharmacol Ther. 20092009 Mar 18. [Epub ahead of print] 
27. Bornhauser M, Schuler U, Porksen G, et al. Mycophenolate mofetil and cyclosporine as graft-versushost disease prophylaxis after allogeneic blood stem cell transplantation. Transplantation 1999;67:499-504. [PubMed: 10071016]

28. Jacobson P, Rogosheske J, Barker JN, et al. Relationship of mycophenolic acid exposure to clinical outcome after hematopoietic cell transplantation. Clin Pharmacol Ther 2005;78:486-500. [PubMed: 16321615]

29. Barker JN, Weisdorf DJ, DeFor TE, Blazar BR, Miller JS, Wagner JE. Rapid and complete donor chimerism in adult recipients of unrelated donor umbilical cord blood transplantation after reducedintensity conditioning. Blood 2003;102:1915-9. [PubMed: 12738676]

30. Woodahl EL, Wang J, Heimfeld S, et al. A novel phenotypic method to determine fludarabine triphosphate accumulation in T-lymphocytes from hematopoietic cell transplantation patients. Cancer Chemother Pharmacol Feb;2009 63:391-401. [PubMed: 18398611]Epub 2008 Apr9

31. Borthakur G, Kantarjian H, Wang X, et al. Treatment of core-binding-factor in acute myelogenous leukemia with fludarabine, cytarabine, and granulocyte colony-stimulating factor results in improved event-free survival. Cancer 2008;113:3181-5. [PubMed: 18932257]

32. Luminari S, Marcheselli L, Sacchi S, et al. Anthracycline-fludarabine-containing regimens with or without rituximab in the treatment of patients with advanced follicular lymphoma. Cancer 2009;115:1906-13. [PubMed: 19248044]

33. McDonald GB, McCune JS, Batchelder A, et al. Metabolism-based cyclophosphamide dosing for hematopoietic cell transplant. Clin Pharmacol Ther 2005;78:298-308. [PubMed: 16153400]

34. Salinger DH, McCune JS, Ren AG, et al. Real-time dose adjustment of cyclophosphamide in a preparative regimen for hematopoietic cell transplant: a Bayesian pharmacokinetic approach. Clin Cancer Res 2006;12:4888-98. [PubMed: 16914577]

35. US DHHS. FDA CDER. CBER. FDA Guidance for Industry on Population Pharmacokinetics. Feb. 1999

36. Salinger, DH.; McCune, JS.; Blough, DK.; Vicini, P. Mean Squared Error as Criterion for Sampling Schedule Optimization for Individual Dose Targeting in IV Busulfan; Abstracts of the Annual Meeting of the Population Approach Group in Europe (PAGE); 2008; Abstr 1413

37. Monteleone, J.; Duffull, SB. Choice of Best Design. In: Kimko, HC.; Duffull, SB., editors. Simulation for Designing Clinical Trials: A Pharmacokinetic-Pharmacodynamic Modeling Perspective. Vol. First edition ed.. Marcel Dekker; New York: 2003. p. 173-200. 

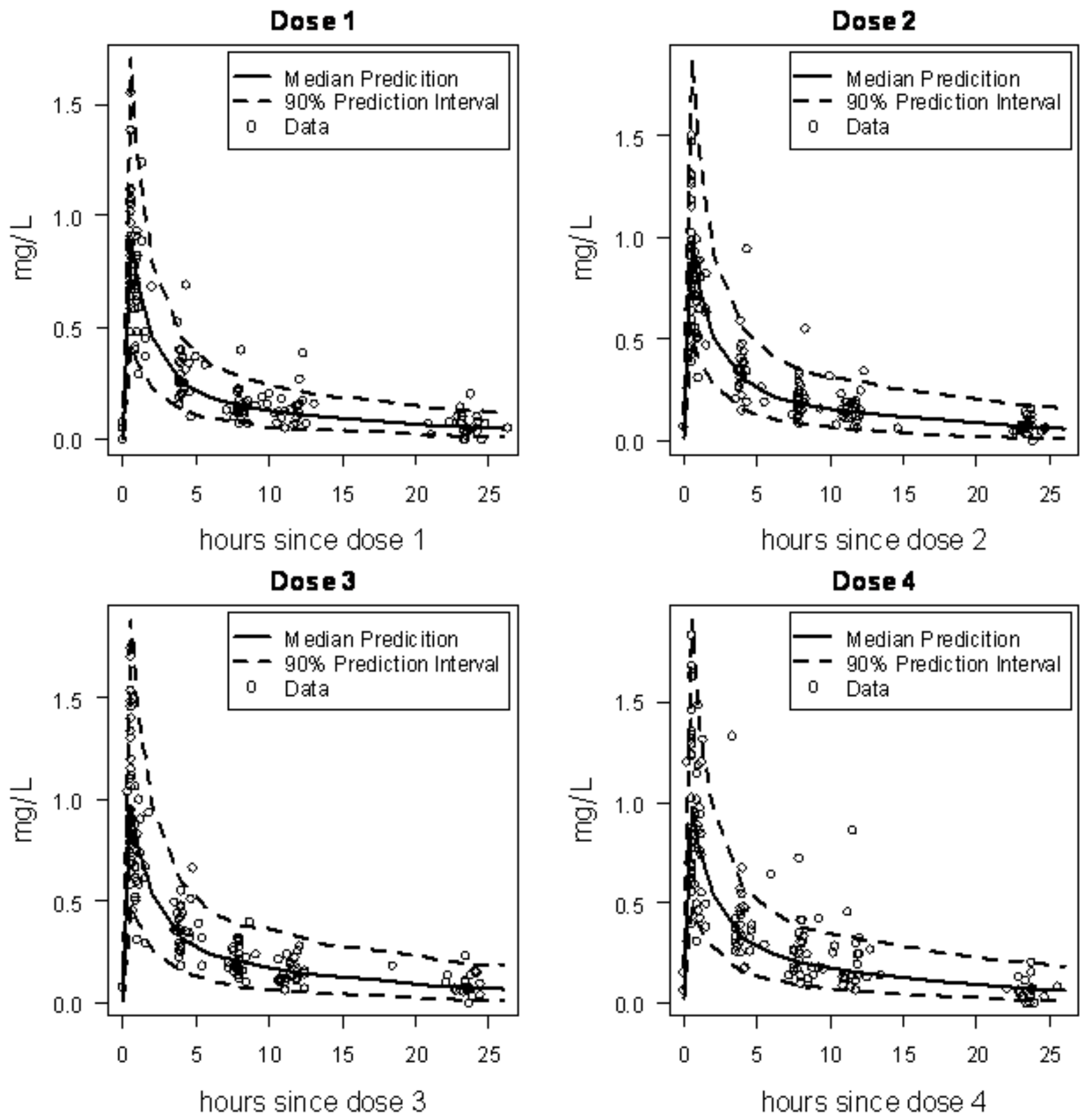

Figure 1.

Visual predictive check for F-ara-A plasma concentrations in HCT patients receiving IV fludarabine $30 \mathrm{mg} / \mathrm{m}^{2} /$ day for 4 days. 
Dose 1

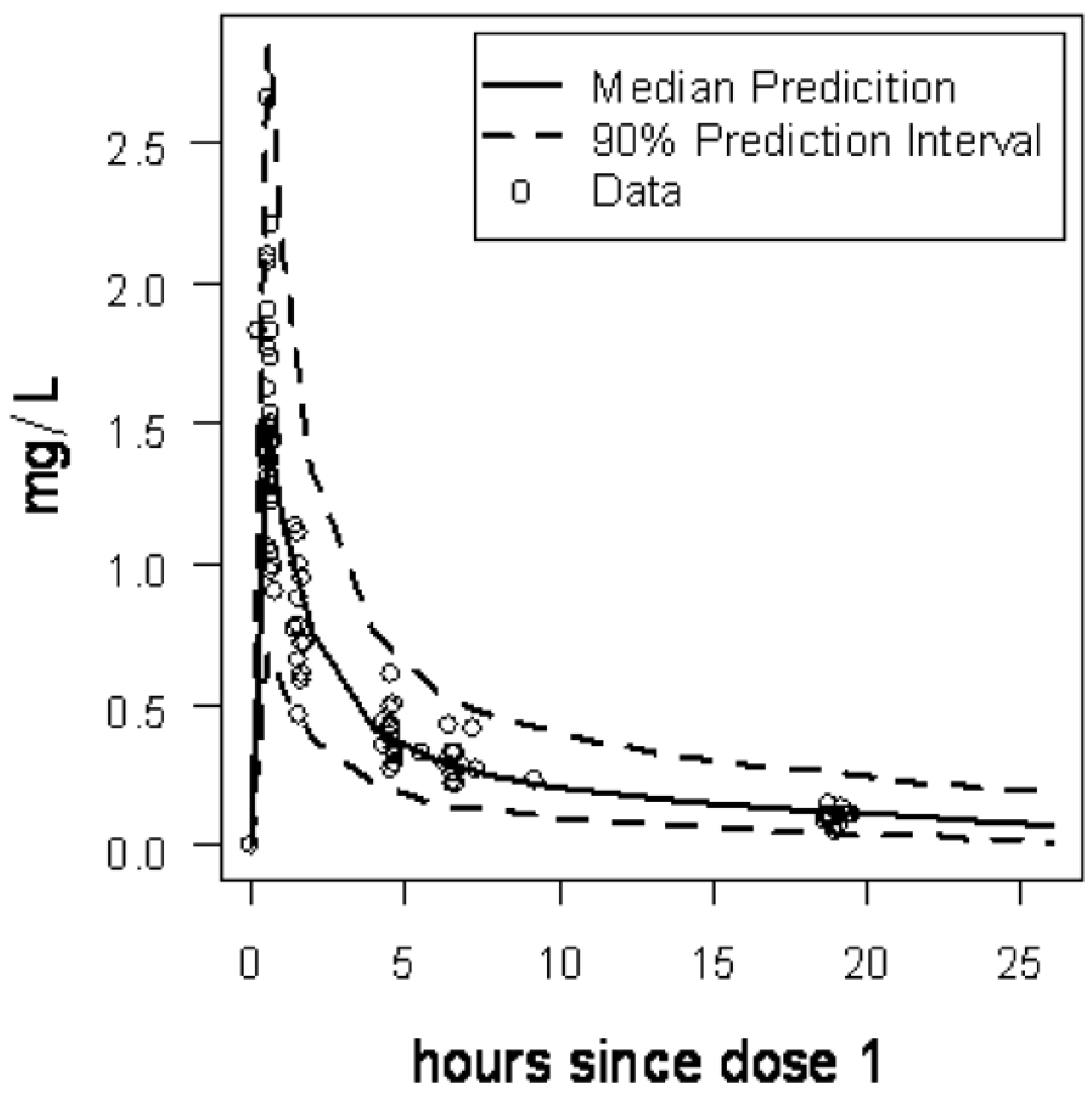

Figure 2.

Visual predictive check for F-ara-A plasma concentrations in HCT patients receiving IV fludarabine $50 \mathrm{mg} / \mathrm{m}^{2} /$ day for 5 days with pharmacokinetic sampling after the first dose only. 


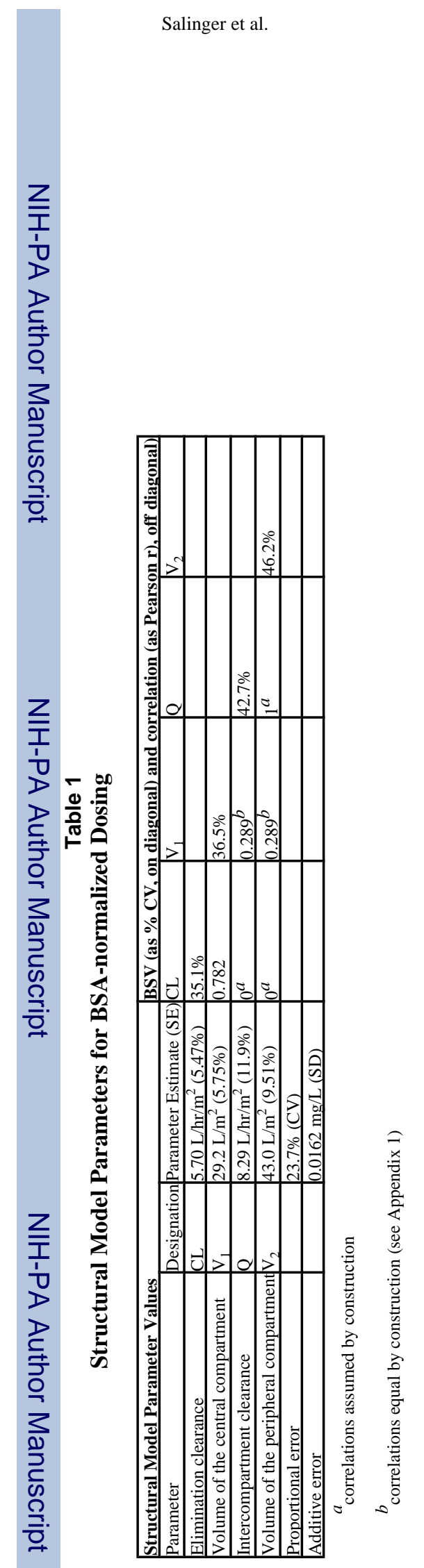

Page 14 


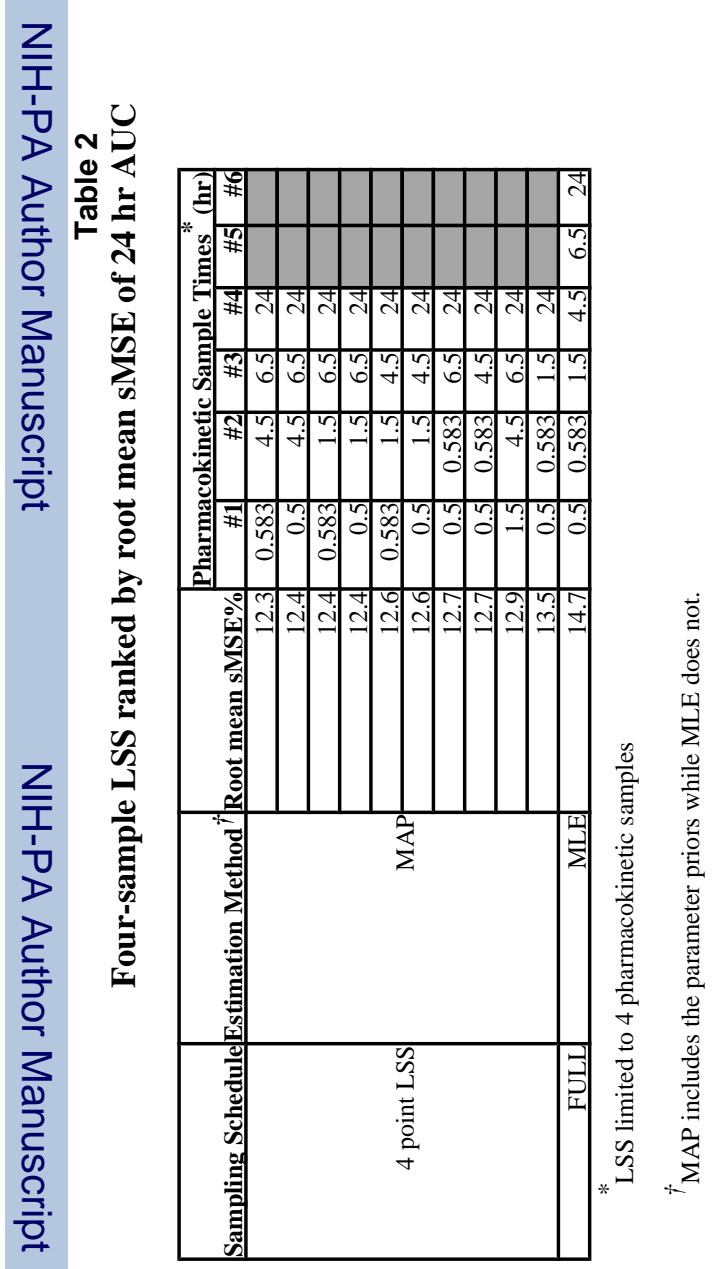

Clin Cancer Res. Author manuscript; available in PMC 2010 August 15. 


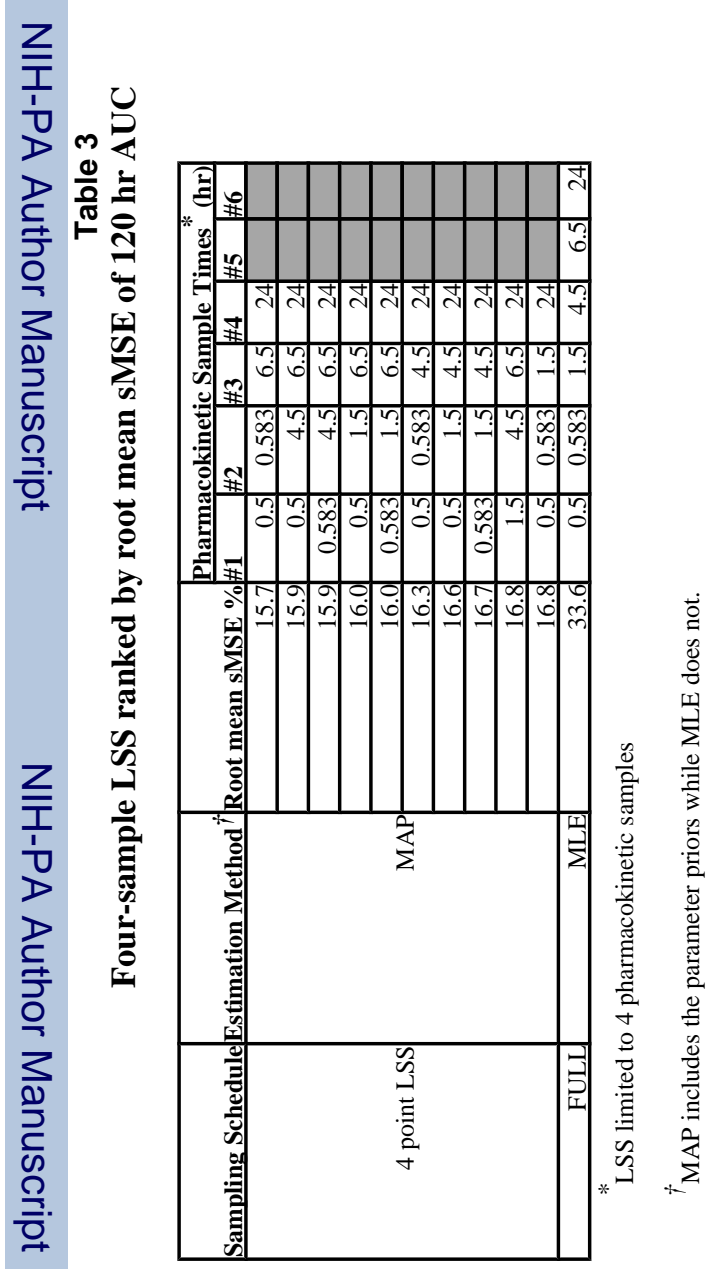

Clin Cancer Res. Author manuscript; available in PMC 2010 August 15. 